\title{
Real-time analysis of gut flora in Entamoeba histolytica infected patients of Northern India
}

\author{
Anil Kumar Verma ${ }^{1}$, Ravi Verma', Vineet Ahuja ${ }^{2}$ and Jaishree Paul ${ }^{*}$
}

\begin{abstract}
Background: Amebic dysentery is caused by the protozoan parasite Entamoeba histolytica and the ingestion of quadrinucleate cyst of E. histolytica from fecally contaminated food or water initiates infection. Excystation occurs in the lumen of small intestine, where motile and potentially invasive trophozoites germinate from cysts. The ability of trophozoites to interact and digest gut bacteria is apparently important for multiplication of the parasite and its pathogenicity; however the contribution of resident bacterial flora is not well understood. We quantified the population of Bacteroides, Bifidobacterium, Ruminococcus, Lactobacillus, Clostridium leptum subgroup, Clostridium coccoides subgroup, Eubacterium, Campylobacter, Methanobrevibacter smithii and Sulphur reducing bacteria using genus specific primers in healthy $(N=22)$ vs amebic patients ( $E$. histolytica positive, $N=17$ ) stool samples by Real-time PCR.

Results: Absolute quantification of Bacteroides $(p=.001)$, Clostridium coccoides subgroup ( $p=0.002)$, Clostridium leptum subgroup ( $p=0.0001)$, Lactobacillus $(p=0.037)$, Campylobacter $(p=0.0014)$ and Eubacterium $(p=0.038)$ show significant drop in their population however, significant increase in Bifdobacterium ( $p=0.009)$ was observed where as the population of Ruminococcus ( $p=0.33$ ) remained unaltered in healthy vs amebic patients (E. histolytica positive). We also report high prevalence of nimE gene in stool samples of both healthy volunteers and amebic patients. No significant decrease in nimE gene copy number was observed before and after the treatment with antiamebic drug.

Conclusions: Our results show significant alteration in predominant gut bacteria in E. histolytica infected individuals. The frequent episodes of intestinal amoebic dysentery thus result in depletion of few predominant genera in gut that may lead to poor digestion and absorption of food in intestine. It further disturbs the homeostasis between gut epithelium and bacterial flora. The decrease in beneficial bacterial population gives way to dysbiosis of gut bacteria which may contribute to final outcome of the disease. Increase in the copy number of nimE gene harboring bacteria in our population reflects possible decrease in the availability of metronidazole drug during treatment of amoebiasis.
\end{abstract}

Keywords: Gut flora, Entamoeba histolytica, RT-PCR

\section{Background}

Entamoeba histolytica, a micro-aerophilic intestinal protozoan parasite and the causative agent of invasive amoebiasis (colitis and amoebic liver abscess), remains a significant cause of morbidity and mortality in developing countries [1]. It is well known that the parasite is constantly interacting with the intestinal gut flora however the contribution of the flora in the manifestation of the disease is poorly understood. The human gastrointestinal

\footnotetext{
* Correspondence: jpaul33@hotmail.com

'School of Life Sciences, Jawaharlal Nehru University, New Delhi, India

Full list of author information is available at the end of the article
}

(GI) tract is nutrient-rich environment packed with a complex and dynamic consortia of trillions of microbes [2].The vast majority reside in our colon where densities approach $10^{11}-10^{12}$ cells $/ \mathrm{ml}$, the highest density recorded for any microbial habitat [3]. About 500-1000 bacterial species colonize the adult intestine,with $30-40$ species comprising up to $97 \%$ of the total population $[4,5]$. Bacteroides, Bifidobacterium, Eubacterium, Clostridium, Peptococcus, Peptostreptococcus, Lactobacillus and Ruminococcus are considered to be predominant genera whereas Enterococcus, Methanobrevibacter and sulphur reducing bacteria (SRB) remain as the subdominant 
genera [6]. The entire system of the human gut microbiota functions as a 'microbial organ' within the intestine, which contributes to diverse mammalian processes including protective functions against pathogens and immunesystem modulation, the metabolic function of fermenting non-digestible dietary fiber, anaerobic metabolism of peptides and proteins that results in the recovery of metabolic energy for the host [7]. The microbial diversity of the human gut is the result of co-evolution between microbial communities and their hosts. Microbial community structure is a very important factor that can influence predisposition to specific diseases in certain host contexts [8].

Ingestion of the cyst of E. histolytica through fecally contaminated food or water initiates infection. Excystation in the intestinal lumen produces trophozoites and colitis results when the trophozoites penetrate the mucus layer and damages intestinal tissues [9]. The trophozoites proliferate in lumen and phagocytose resident flora. E. histolytica trophozoites are quite selective in respect to their interactions with different bacterial species and only those bacteria which have the appropriate recognition molecules get attached and ingested [10]. It has been observed that the nuclear DNA content of $E$. histolytica trophozoites growing in axenic cultures is at least 10 fold higher than in xenic cultures and re-association of axenic cultures with their bacterial flora led to a reduction of DNA content attaining the original xenic values indicating a flexible nature of the parasite genome [11]. Fluctuations in gut flora have been reported both in acute diarrhea and antibiotic associated diarrhea [12], but very few reports are available on status of gut flora in E. histolytica infected individuals. Earlier studies in our laboratory [1] have recorded fluctuations in the gut flora by a qualitative method during disease conditions.

5-Nitroimidazole drugs are still used as first line of defense against amoebic and other infections caused by anaerobes. These drugs are administered as pro drugs and one electron reduction of nitro group converts the pro drug into an active drug [13]. Enzymatic modification mediated by nim-class of genes is a well characterized resistance mechanism. Certain Bacteroides species which are members of the normal colonic human microflora harbor nim genes [14]. Our study is based on the hypothesis that the Entamoeba histolytica (but not E. dispar) is an invasive organism and invades the mucus layer and subsequently the intestinal epithelium for colonization using the pathogenic factors. In this context we attempted to study the fluctuations in the gut microbiota that contributes to substantial metabolic changes in E. histolytica infected individuals compared to healthy individuals. In the present study we used Real Time PCR for absolute quantification of predominant gut bacterial population in E. histolytica patients suffering from dysentery for 5-7 days. We also quantified the copy number of nim gene in stool sample of healthy vs $E$. histolytica patients.

\section{Methods}

\section{Study subjects $\&$ fecal sample collection}

Stool samples of healthy person (without any enteric disease) were collected as controls from volunteers of a community in Delhi. Initial survey involved discussion with the focus group and informed consent was taken from participating volunteers for the study. Volunteers in age group of 21-40 year (mean age 31 year) were randomly recruited. Subjects who have taken any antibiotic/ antiamoebic drug or suffered from any gastrointestinal disorder in past one month before sample collection were not included in the study. Twenty two stool samples were collected from healthy volunteers. Clinical diagnosis of amoebic colitis was based on standard criteria: patients experiencing days to weeks of dysentery (stool with blood and mucus) or diarrhea with cramps followed by abdominal pain and/or weight loss. The sub acute onset of the disease was a helpful clue in the differential diagnosis because bacillary dysentery caused by Shigella, Salmonella, Campylobacter and EHEC E. coli mostly lead to a abrupt onset of the disease [15]. Since we did not take samples from individuals administered with any antibiotic, therefore cases of antibiotic associated diarrhea were excluded. Stool samples of chronic/ acute diarrhea as diagnosed by Gastroenterologist were collected from Gastroenterology department of All India Institute of Medical Sciences \& Safdarjung hospitals, New Delhi. The samples were transported to the laboratory at $4^{\circ} \mathrm{C}$ within $2 \mathrm{hrs}$ and stored at $-20^{\circ} \mathrm{C}$ until processed. The study was approved by the research ethics board of respective institutes. The samples $(\mathrm{n}=550)$ were collected with the informed consent of the patients.

\section{Enrichment of entamoeba cysts}

Cysts were enriched following the protocol of Knight et al., 1976 [16] with slight modifications. Briefly, fecal samples $(1 \mathrm{gm})$ were homogenized in $10 \mathrm{ml}$ of autoclaved distilled water, strained through cheesecloth in $50 \mathrm{ml}$ falcon tube. This suspension was centrifuged at $2000 \mathrm{rpm}$ for $5 \mathrm{~min}$ and pellet was re-dissolved in $10 \mathrm{ml}$ of $10 \%$ formaldehyde. $3 \mathrm{ml}$ of diethyl ether was added to the tube and this mixture was vortexed and incubated at RT for $30 \mathrm{~min}$. The mixture was subjected to centrifugation at $2000 \mathrm{rpm}$ for $5 \mathrm{~min}$, supernatant was removed and pellet was washed with double distilled water. The Pellet containing concentrated cyst was re-dissolved in $400 \mu \mathrm{l} \mathrm{T}{ }_{10} \mathrm{E}_{1}$ buffer. Cysts in $\mathrm{T}_{10} \mathrm{E}_{1}$ buffer was subjected to freeze-thaw cycle and thereafter to sonication in order to obtain crude DNA for Dot-blot hybridization experiment. 


\section{Screening of samples by dot blot hybridization}

The crude cyst DNA was denatured by addition of $\mathrm{NaOH}$ to final concentration of $0.25 \mathrm{~N}$ in a total volume of $300 \mu \mathrm{l}$. The DNA was kept at room temperature for 30 minutes and then transferred on to ice. The GS + nylon membrane of required size was cut and saturated in $0.4 \mathrm{M}$ Tris- $\mathrm{Cl}, \mathrm{pH} 7.5$ for $15 \mathrm{~min}$ and the DNA were spotted on to the membrane with the help of mini-fold apparatus from Whatman, Germany. The blots were air dried and UV cross linked before hybridization. We used $4.5 \mathrm{~kb}$ rDNA fragment (EcoRI to Hind III site) from HMe region of EhR1 (rDNA plasmid in HM1:IMSS strain of E.histolytica) as probe for detection of Entamoeba positive samples that include both E.histolytica and $E$. dispar (Figure 1A) [17].

\section{Genomic DNA extraction}

DNA was extracted from the Dot blot positive samples. An aliquot of $200 \mathrm{mg}$ stool sample was used for isolation using QIAamp mini stool kit (QIAGEN,Germany) as per manufacturer's guidelines. While isolating DNA from the stool samples through the above kit, pGEMT-easy plasmid containg $240 \mathrm{bp}$ fragment of glycoprotein $B(\mathrm{gB})$ gene of phocine virus (20 ng/200 $\mu \mathrm{l}$ of ASL buffer) was added in ASL buffer as internal control during the isolation of genomic DNA [18].

\section{PCR analysis of Dot blot positive samples}

To differentiate Dot-blot positive samples into E. histolytica and $E$. dispar, primers were designed from EhSINE2 for $E$. histolytica and from $18 \mathrm{~S}$ and ITS2 region of rDNA circle for $E$. dispar respectively (Figure 2A \& B). Primer sequences were as follows; Eh-F 5'-GTCAGAGACACCACATGAA-3, Eh-R 5'-GAGACCCCTTAAAGAAAC -CC-3' and EdF 5'-GAAGAAACATTGTTTCTAAATCCAA-3' \& Ed-R 5'-TTTATTAA CTC ACTTATA-3' [19].

\section{Primer designing for detection of predominant genera of gut flora}

Primer sets were designed to differentiate and quantitate the following major anaerobic genera-Bacteroides, Clostridium, Campylobacter, Bifidobacterium, Ruminococcus, Eubacterium, Lactobacillus, Methanobrevibacter and Sulfate-reducing bacteria (SRB).16S rRNA gene was targeted for designing primers except for SRB (Table 1). Sulphate reducing gene was targeted for quantifying members of SRB. Primers were commercially obtained from Sigma-aldrich, USA.

A.

$\mathrm{HMe}(\mathrm{E}+\mathrm{H})$

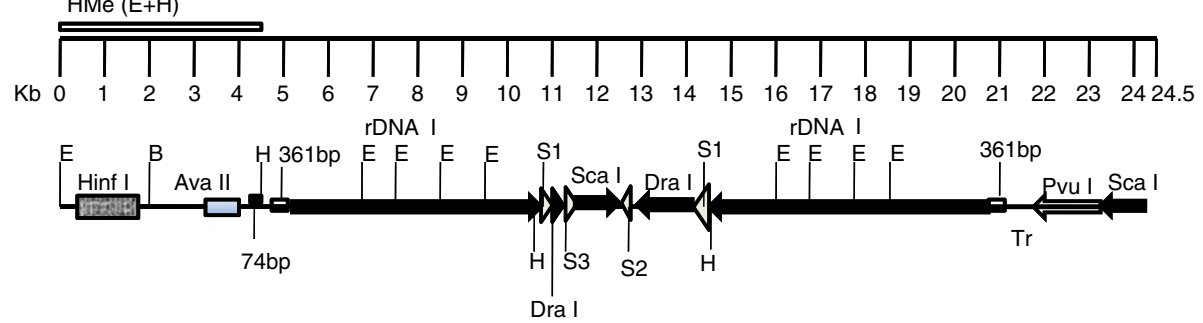

B.

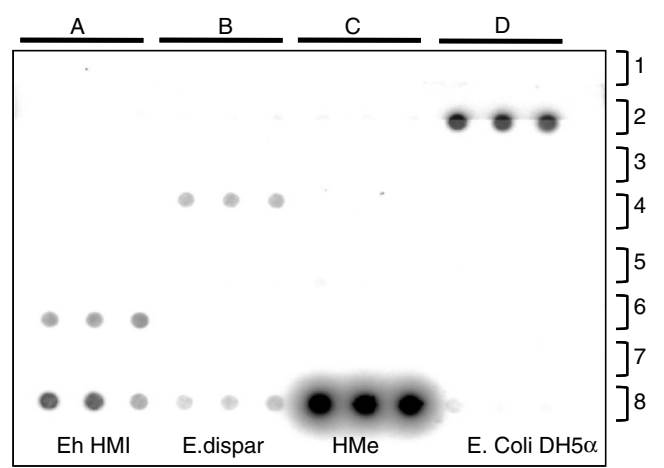

Figure 1 Screening of stool samples by Dot-Blot method. (A) Linear map of EhRI episome (24.5 kb) showing the position of HMe probe (4.5 $\mathrm{kb}$ in size) common for both E. histolytica and E. dispar), E - EcoR1 site and H- Hind III site; rDNA I and rDNA II represent two inverted repeats of transcription units with various restriction sites and repeats (B) Representative figure of Dot-blot analysis of stool sample using HMe probe. Rows 1 to 6 (column A-D) represent spots of DNA from stool samples. About 20 ng of DNA was loaded on each spot in triplicate on nylon membrane. Row 7 was blank. Row 8 (column A) E. histolytica HM1: IMSS genomic DNA as positive control; (column B) E. dispar SAW760 genomic DNA as positive control; (column C) E.Coli DH5a as negative control; (column D) Plasmid with cloned HMe as positive control. All samples were loaded in triplicate. Experimental details are provided in material and methods. 
A.

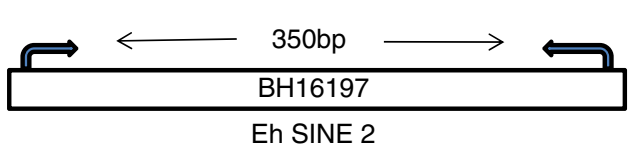

C.

PCR with E. histolytica specific primer

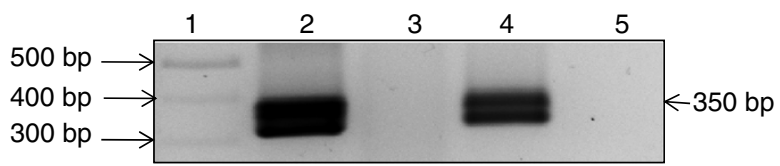

B.

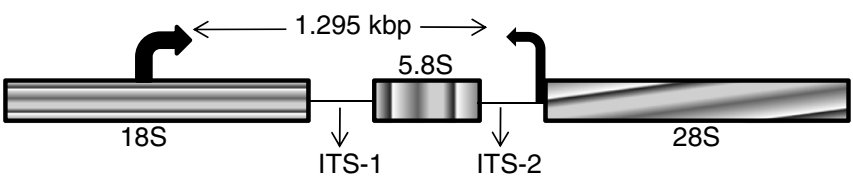

D.

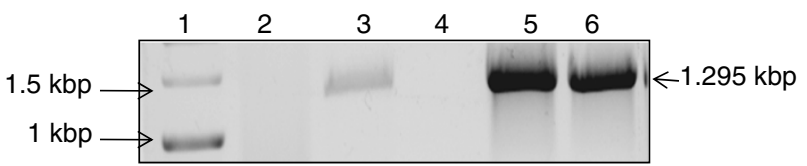

Figure 2 Screening of Stool samples by PCR. (A) Schematic representation of location of Entamoeba histolytica specific primer. BH16197 is Genbank accession number of Entamoeba histolytica SINE-2 (EhSINE2) element; (B) Schematic representation of location of Entamoeba dispar specific primer from rDNA molecule. $18 \mathrm{~S}, 5.8 \mathrm{~S}$ and $28 \mathrm{~S}$ are corresponding ribosomal gene sequences and ITS-1 and ITS-2 refers to internal transcribed spacer 1 and 2; (C) Detection of E. histolytica in stool DNA sample using E. histolytica specific primers, Lane $1=$ Marker 100 bp, Lane 2 $=$ EhHM1 genomic DNA as positive control, Lane 3 \& 4 stool sample DNA, Lane $5=$ Genomic DNA of E. dispar SAW760 as negative control. Sample in lane 4 is E. histolytica positive; (D) Detection of E. dispar in Stool sample using E. dispar specific primers. Lane $1=$ Marker $1 \mathrm{~kb}$, Lane 2,3,4 and 5 stool sample DNA, Lane $6=$ Genomic DNA of E. dispar as positive control. Sample in Lane 3 and 5 are E. dispar positive. Lane 4 stool sample is $E$. histolytica positive and was used as negative control.

Table 1 Genus specific 16S rRNA targeted bacterial primers used in this study

\begin{tabular}{|c|c|c|c|c|c|}
\hline Sr no. & Genus & Primer sequence & PCR Product (bp) & $\operatorname{Tm}\left({ }^{\circ} \mathrm{C}\right)$ & References \\
\hline \multirow[t]{2}{*}{1.} & Methanobrevibactr & F 5'- CGATGCGGACTTGGTGTTG-3' & 184 & 59.7 & [21] \\
\hline & & R 5'-TGTCGCCTCTGGTGAGATGTC-3' & & 59.8 & \\
\hline \multirow[t]{2}{*}{2.} & Peptostreptococcus & F 5'-AACTCCGGTGGTATCAGATG-3' & 270 & 55.4 & [1] \\
\hline & & R 5'-GGGGCTTCTGAGTCAGGTA-3' & & 56.4 & \\
\hline \multirow[t]{2}{*}{3.} & Ruminococcus & F 5'-GAAAGCGTGGGGAGCAAACAGG-3' & 302 & 65.8 & [21] \\
\hline & & R 5'- GACGACAACCATGCACCACCTG-3' & & 64.4 & \\
\hline \multirow[t]{2}{*}{4.} & Eubacterium & F 5'-GTAGTCCACGCCGTAAACGATG-3' & 278 & 60.4 & [21] \\
\hline & & R 5'-ACACGAGCTGACGACAACCATG-3' & & 62.4 & \\
\hline \multirow[t]{2}{*}{5.} & Bacteroides & F 5'- GGGGTTCTGAGAGGAAG-3' & 115 & 54.0 & [21] \\
\hline & & R 5'- GCTACTTGGCTGGTTCAG-3' & & 56.0 & \\
\hline \multirow[t]{2}{*}{6.} & Lactobacillus & F 5'-GCAGCAGTAGGGAATCTTCCA-3' & 340 & 64.0 & [25] \\
\hline & & R 5'-GCATTYCACCGCTACACATG-3' & & 58.0 & \\
\hline \multirow[t]{2}{*}{7.} & Clostridium leptum subgroup & F 5'-CGTCAGCTCGTGTCGTGAGAT-3' & 125 & 60.0 & [21] \\
\hline & & R 5'-CGTCATCCCCACCTTCCTCC-3' & & 62.5 & \\
\hline \multirow[t]{2}{*}{8.} & Clostridium coccoides subgroup & F 5'-GCCACATTGGGACTGAGA-3' & 170 & 56.0 & This study \\
\hline & & R 5'-GCTTCTTAGTCAGGTACCG-3' & & 58.0 & \\
\hline \multirow[t]{2}{*}{9.} & Campylobacter & F 5'-AGGGAATATTGCGCAATGGGGGAAA-3' & 180 & 58.0 & [21] \\
\hline & & R 5'- GATTCCGAGTAACGCTTGCACCCT-3' & & 59.0 & \\
\hline \multirow[t]{2}{*}{10.} & Bifidobacterium & F 5'-GATTCTGGCTCAGGATGAACGC-3' & 231 & 61.9 & [21] \\
\hline & & R 5'-CTGATAGGACGCGACCCCAT-3' & & 60.8 & \\
\hline \multirow[t]{2}{*}{11.} & Sulfate-reducing bacteria (APS reductase & F 5'-TGGCAGATMATGATYMACGG-3' & 396 & 54. & This study \\
\hline & subunit A gene) & R 5'-GGCCGTAACCGTCCTTGAA-3' & & 54.0 & \\
\hline
\end{tabular}




\section{Primers for detection and quantification of nim gene}

Primers were designed from nim gene after Stephanie Trinh et al. [14]. Primer sequences were as follows; NIM-F (5'-ATGTTCAGAGAAATGCGGCGTAAGCG-3') and NIM-R (5'-GCTTCCTTGCCTGTCAT GTGCTC-3'). Primers Nim-F and Nim-R designed by us amplify all the members of nim gene family viz. $\operatorname{nim} A, \operatorname{nim} B, \operatorname{nim} C$, nimD and nimE. Primers were commercially synthesized from Sigma-Aldrich, USA. Primers NIM-F\&R did not amplify genomic DNA derived from axenic culture of E. histolytica HM1-IMSS [1] and blast result of the selected primers did not show any homology with E. histolytica genome. NIM-F\&R primers amplified $458 \mathrm{bp}$ fragment of nim gene from stool sample DNA. This amplified fragment of $458 \mathrm{bp}$ was cloned in pGEMT-easy vector and sequenced to ensure the amplification of correct gene. The clone was subsequently used as a standard for quantification of nim gene by Real Time-PCR.

\section{PCR-RFLP of nim gene}

Primers NIM-F and NIM-R were used to amplify all the members of nim gene family from stool sample DNA. Members of nim gene family were differentiated by digesting the PCR product with restriction enzymes HpaII and TaqI. HpaII digests nimA, nimC, nimD at different loci but not nimB and nimE where as TaqI digests $\operatorname{nim} A, \operatorname{nim} B, \operatorname{nim} E$ at different loci but not nim $C$ and nimD [19].

\section{Reference strains}

Genus specific primers were used to amplify respected genera from DNA of stool sample of healthy individual. The amplified product was cloned and sequenced and sequences were deposited in EMBL database to obtain the accession numbers (Table 2). These $16 \mathrm{~S}$ rRNA gene fragment containing plasmids were used as reference strains.

\begin{tabular}{|c|c|c|}
\hline Bacteria & Source & Accession no. \\
\hline Bacteroides & Stool of healthy individual & AM117604 \\
\hline Methanobrevibacter & Stool of healthy individual & FN813615 \\
\hline Eubacterium & Stool of healthy individual & FN813614 \\
\hline Lactobacillus & Stool of healthy individual & AM042701 \\
\hline Bifidobacterium & Stool of healthy individual & AM042698 \\
\hline Clostridium & Stool of healthy individual & AM042697 \\
\hline Campylobacter & Stool of healthy individual & AM042699 \\
\hline Ruminococcus & Stool of healthy individual & FN823053 \\
\hline Sulfate-reducing bacteria & Stool of healthy individual & FN995351 \\
\hline
\end{tabular}

\section{Real time PCR analysis of bacterial population}

Quantification was done using ABI-7500 machine and power syber green PCR master mix kit from Applied Biosystems, USA. Standard curve was the method of choice for absolute quantification of bacteria. Standard curve was made using serial dilutions of plasmid (containing $16 \mathrm{~S}$ rRNA gene fragment) of known concentrations on tenfold basis. With the molecular weight of the plasmid and insert known, it is possible to calculate the copy number as follows:

Step 1: Determining molecular weight (mw)

Weight in Daltons $(\mathrm{g} / \mathrm{mol})=(\mathrm{bp}$ size of double stranded product)(330 Da $x$ 2nt/bp)

Step 2: Molecular weight to copy number

$\mathrm{X}$ g/mol/Avogadro's number $\left(6.023 \times 10^{23}\right.$ molecules $/$ mole $)=X \mathrm{~g} /$ molecule Where $\mathrm{X}=$ the weight of one molecule or copy Where $\mathrm{bp}=$ base pairs, $\mathrm{nt}=$ nucleotides [20]

Real time PCR runs were performed in 96 well optical plates (each containing 1x PCR master mix, $4 \mathrm{pm} / \mu \mathrm{l}$ forward and reverse primer(optimized concentration) and $1 \mu \mathrm{l}$ plasmid DNA of tenfold dilutions or $1 \mu \mathrm{l}$ DNA from samples in $20 \mu$ l reaction) for 40 cycles using an $\mathrm{ABI}$ 7500 sequence detector (Applied biosystems). Default 7500 cycle conditions were used with only change in the annealing temperature. A standard curve was drawn by plotting the natural log of the threshold cycle $(\mathrm{Ct})$ against the natural $\log$ of the number of molecules. Melting curves were obtained from $55^{\circ} \mathrm{C}$ to $90^{\circ} \mathrm{C}$, with fluorescence measurements taken at every $1^{\circ} \mathrm{C}$ increase in temperature. All reactions were carried out in triplicate along with a non-template control. Ct values were calculated under default settings for the absolute quantification using the software provided with the instrument. The equation drawn from the graph was used to calculate the precise number of target molecule (plasmid copy no. or number of bacteria) tested in same reaction plate as standard as well as in sample.

\section{Statistical analysis}

Graph of respective bacterial population is plotted as mean value with standard error. Each sample was analyzed in triplicate for calculation of significant differences in bacterial population by the Man-Whitney test. P values of 0.05 or below considered as significant. Paired samples collected from healthy volunteers before and after satronidazole treatment were analyzed by Wilcoxon matched- 
pairs signed rank test (two tailed). Analysis was done using GraphPad Prism-5 software.

\section{Results}

\section{Screening of E. histolytica positive samples}

DNA from concentrated cyst was subjected to Dot-blot hybridization. Dot blot analysis of 550 samples yielded 39 samples (7\%) that were positive for Entamoeba (Figure 1B). The DNA from Entamoeba positive samples were subjected to PCR using species specific primers of E. histolytica and E. dispar (Figure 2C \& D). Out of 39 samples, 17 samples (43\%) were positive for E. histolytica. None of the samples in our study population were found positive for both the species of the parasite.

\section{Quantification of predominant flora}

High quality DNA isolated from $E$. histolytica positive stool sample was subjected to Real Time analysis to assess the predominant gut flora that included Bacteroides, Bifidobacterium, Eubacterium, Clostridium leptum subgroup, Clostridium coccoides subgroup, Lactobacillus and Ruminococcus. Two subdominant genera Methanobrevibacter smithii and Sulphur reducing bacteria (SRB) were also quantified. Validation of primers designed by us for the above genera have already been reported [21]. In addition to the above primers, here we report a Real time analysis of nim gene copy number for which a standard curve and amplification curve have been drawn that shows specific and efficient quantification with slope $=-3.6$ and $\mathrm{R}^{2}=0.998$ (Figure 3A \& B).

Our analysis reveals that during healthy conditions, the members of Bacteroides were the most abundant in number among the predominant targeted genera. However, a significant decrease was observed in population of Bacteroides $(\mathrm{p}=.001)$ in E. histolytica positive samples when compared to that of Healthy control samples (Figure 4A). Simultaneously, we also observed a significant decrease in the population of Closrtridium coccoides subgroup $(\mathrm{p}=0.002)$, Clostridium leptum subgroup $(\mathrm{p}=0.0001)$, Lactobacillus $(\mathrm{p}=0.037)$, Campylobacter $(\mathrm{p}=0.0014)$ and Eubacterium $(\mathrm{p}=0.038)$ in E. histolytica positive samples in comparison to control (Figure 4B, C, D, E and F respectively). Surprisingly, we observed a significant rise in the population of Bifidobacterium $(\mathrm{p}=0.009)$ in amebic samples when
A.

Standard curve

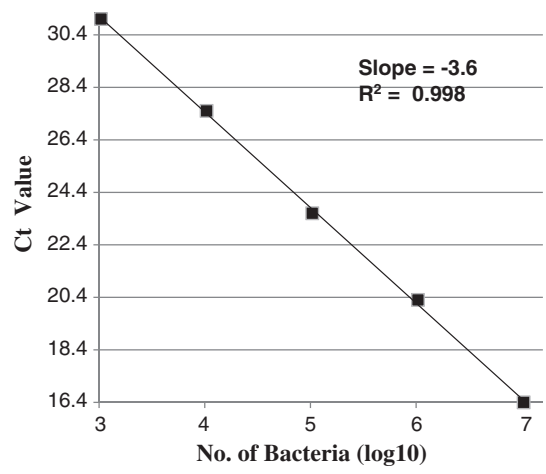

C.

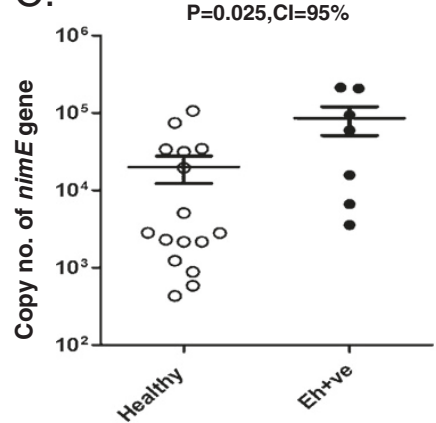

B.

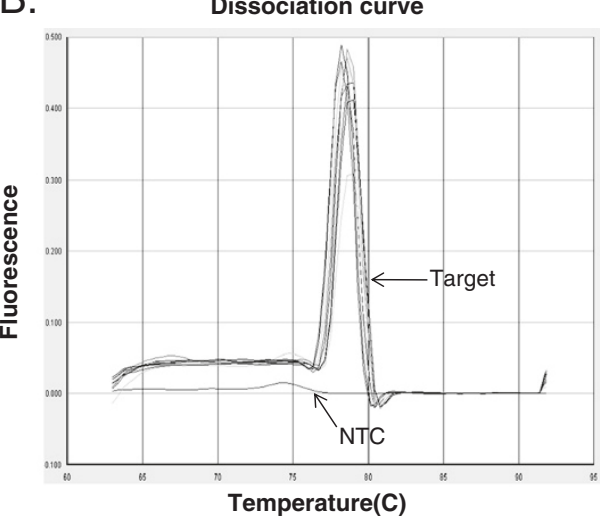

D.

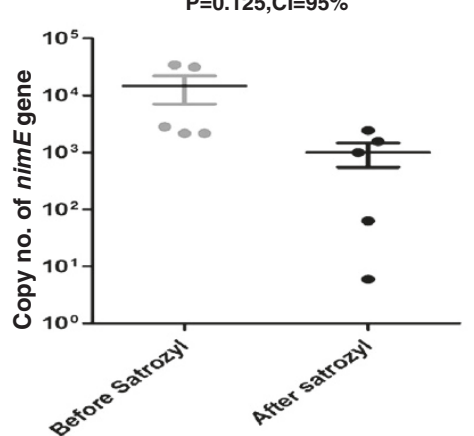

Figure 3 Detemination of copy number of nimE gene by Real-time PCR. (A) Standard curve, slope $=-3.6$ and $R^{2}=0.998$ showing good efficiency. (B) Dissociation curve showing specific amplification of target (nimE gene) and NTC = No template control. (C) Absolute quantification of copy no. of nimE gene in Healthy vs E. histolytica positive samples. (D) Absolute quantification of copy no. of nimE gene in stool sample DNA of Healthy volunteers before and after satronidazole treatment. P value $=.05$ or below was considered significant. Cl stands for confidence interval. 


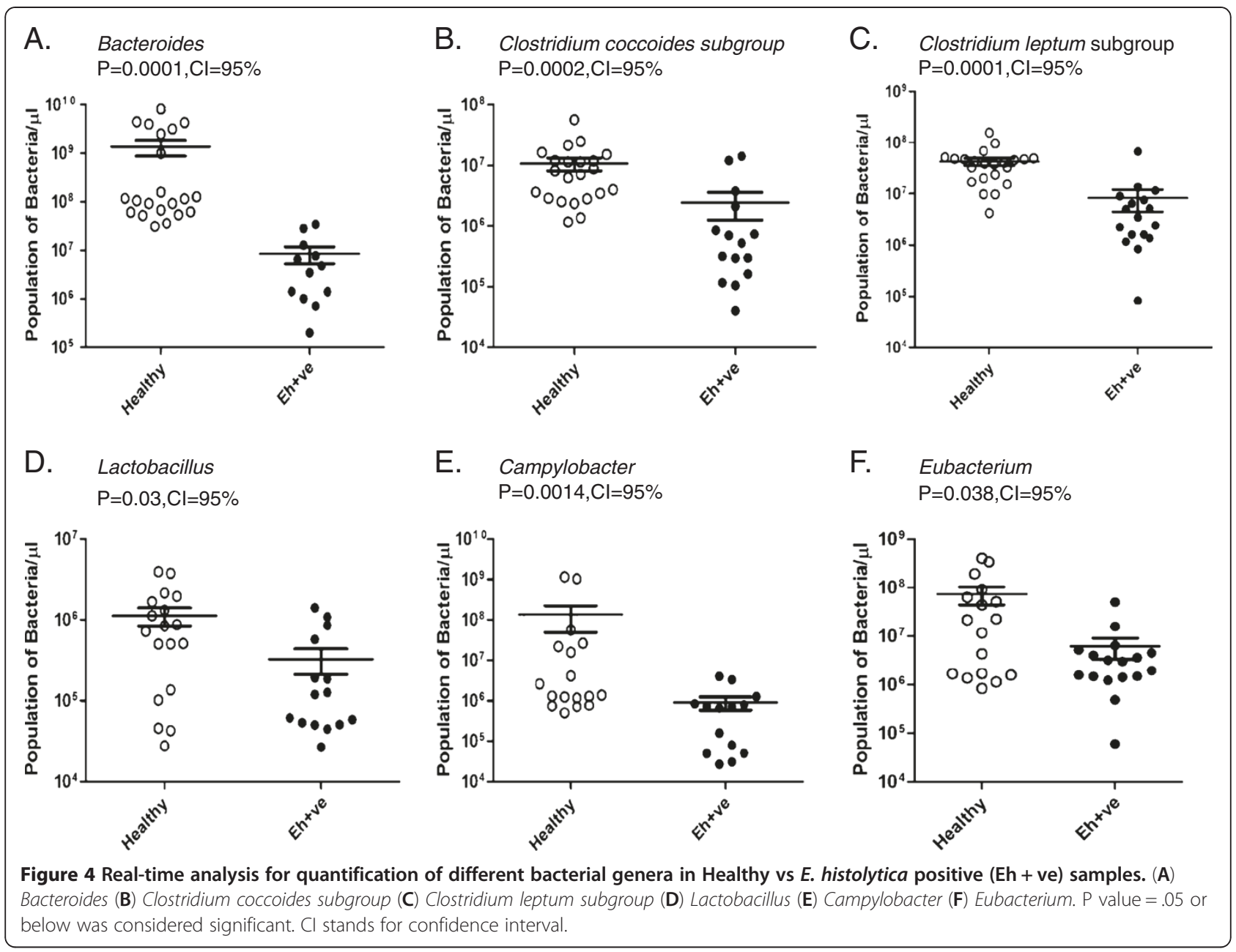

compared with healthy control samples (Figure 5B). No significant changes were observed in population of Rumminococcus $(\mathrm{p}=0.33)$ (Figure $5 \mathrm{~A})$. Though we did not observe any significant change in the population of Methanobrevibacter $(\mathrm{p}=0.96)$ and Sulphur reducing bacteria $(\mathrm{p}=0.88)$ in amoebic samples but the prevalence rate was reduced (Additional file 1: Figure S1A \& B).

\section{Copy no. of nim gene}

We found the presence of nim genes in $72.7 \%$ of control stool samples $(\mathrm{n}=22)$ and in $41 \%$ of Entamoeba histolytica infected patients $(\mathrm{n}=17)$ by PCR (Figure 6A). Further the amplified product was cloned and sequenced. BLAST analysis revealed $99 \%$ sequence homology with nimE gene (Accession no. AM117602.1), a member of nim gene family [22]. Subsequently, the PCR products from all the samples of healthy and amebic individuals were subjected to RFLP analysis using HpaII and TaqI restriction enzymes. PCR-RFLP pattern confirmed the presence of only nimE gene in all the samples analyzed (Figure 6B \& C). Real time analysis of nim gene in the stool samples exhibited sample to sample variation $\left(4 \times 10^{2}\right.$ to $4 \times 10^{5}$ copies $)$ in the both category of samples. We observed a significant increase in copy no. of nim gene in E. histolytica positive samples vs samples from healthy persons $(\mathrm{p}=0.025)$ (Figure 3C).

To see the effect of antiamoebic drug Satronidazole (Alchem pharmaceuticals) on nim gene copy number, healthy volunteers $(n=5)$ were advised to take the drug (300 mg tablets) twice daily after meals for 4 days and copy of nim gene was quantified before and after the treatment using the primers described here. Wilcoxon matched-pairs signed rank test (two tailed) analysis of copy no. of nim gene shows no significant change $(\mathrm{p}=0.125)$ in stool samples collected before and after treatment (Figure 3D).

\section{Discussion}

Infection by E. histolytica is normally initiated by the ingestion of fecally contaminated water or food containing E. histolytica cysts. Phagocytosis of colonic bacteria has been considered as a possible stimulus to induce the invasive behavior by the parasite [23]. Adult gut microbiota are quite stable in individuals and can even be restored after perturbation [24,25]. Our earlier results 


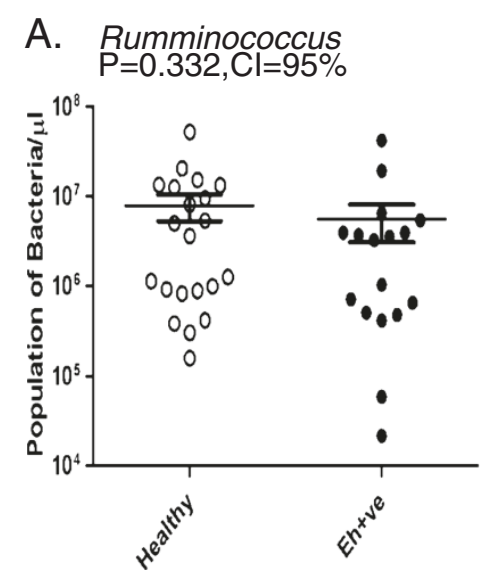

\section{B. Bifidobacterium}

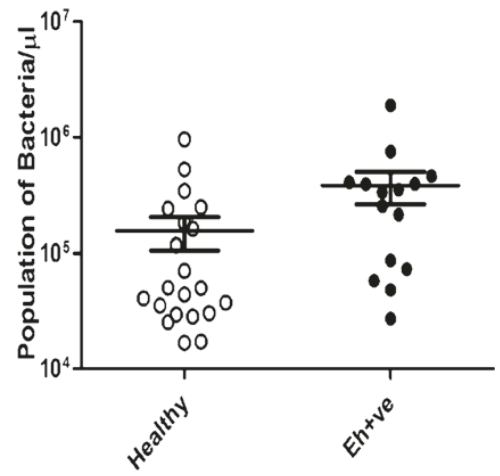

Figure 5 Real-time analysis of population of (A) Rumminococcus in Healthy vs E. histolytica positive (Eh + ve) samples (B) Bifidobacterium in Healthy vs $E$. histolytica positive $(\mathrm{Eh}+\mathrm{ve})$ samples. $\mathrm{P}$ value $=.05$ or below was considered significant. $\mathrm{Cl}$ stands for confidence interval.

have shown significant changes in expression of EhCaBP and LPG only after the axenic E. histolytica had been adapted to grow with bacterial flora for a number of generatiom, and not in short term culture [26]. In the present study we tried to evaluate perturbations in commensal gut flora caused as result of E. histolytica infection using Real Time PCR. qPCR methodology is less expensive, more quantitative and is more efficient in terms of time and operation [27]. The absolute proportions of eight predominant commensal and two subdominant genera were quantified successfully in our samples.

Bacteroides species are a pleomorphic group of non-spore forming gram-negative anaerobic bacteria. Bacteroides are the most dominant part of the normal indigenous flora in the human gut. Bacteroides are mostly represented by Bacteroides ovatus, Bacteroides uniformis Bacteroides vulgatus, Bacteroides thetaiotaomicron, Bacteroides distasonis, and less frequently by Bacteroides eggerthii and Bacteroides fragilis. These bacteria are significant contributors to the carbohydrate metabolism, nutrition and health of humans and animals. In 1999 Hooper et al. demonstrated that B. thetaiotaomicron can modify intestinal fucosylation in a complex interaction mediated by fucose repressor gene and a signaling system [28]. The significant decrease in population of Bacteroides during disease condition dampens the beneficial effects of this genera to host.

In the human intestinal tract, Eubacterium is the second most common genus after the genus Bacteroides. The genus Eubacterium comprises a nutritionally diverse group of organisms. The members of genus Eubacterium are known to produce butyrate [29], degrade flavonoids (from vegetables, fruits, nuts, and tea) [30] and are implicated in steroid and bile transformation in intestine
[31]. The decrease in population of Eubacterium sp. observed in our study may reduce the butyrate production and may also affect the capacity of the host in proper digestion of the above ingredients of food.

Bifidobacterium species are common inhabitants of the gastrointestinal tract, and they have received special attention because of their health-promoting effects in humans. Members of Bifidobacteria produce enough acetate (SCFA) in proximal and distal colon by fermentation of glucose and fructose [32]. Members of both Bifidobacteria and Ruminococcus -Ruminococcus torques and Bifidobacterium bifidum are thought to ferment mucin and compete to colonise this substrate for their energy source [33]. Our result shows a significant increase in population of Bifidobacterium but no change in population of Rumminococcous despite decrease in population of several other targeted genera. It is quite well known that mucus secretion is increased in E. histolytica infection especially during dysentery which is probably result of a mechanism exerted by intestinal epithelial cells to counter the adherence of E. histolytica trophozoites to intestinal epithelial surface. The protozoan parasite Entamoeba histolytica cleaves Mucin 2 (MUC2) in the non-glycosylated oligomerization domains by cysteine protease, thus breaking down the macromolecular structure and reducing mucus viscosity [34]. Perhaps under this condition, a cross-talk between the mucosal layer, bacteria and the parasite initiates. As a result, the intestinal epithelial cells tend to produce more of mucin for protection that promotes colonization of Bifidobacteria in one hand and on the other hand the parasite competes to more release of mucin for its adhesion to epithelial layer. Bifidobacteria longum are known to protect the gut from enteropathogenic infection through production of acetate [32] and acetate is major energy source for colonocytes but a fine 


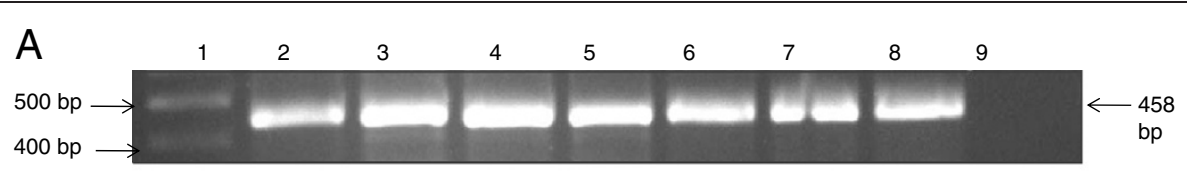

B
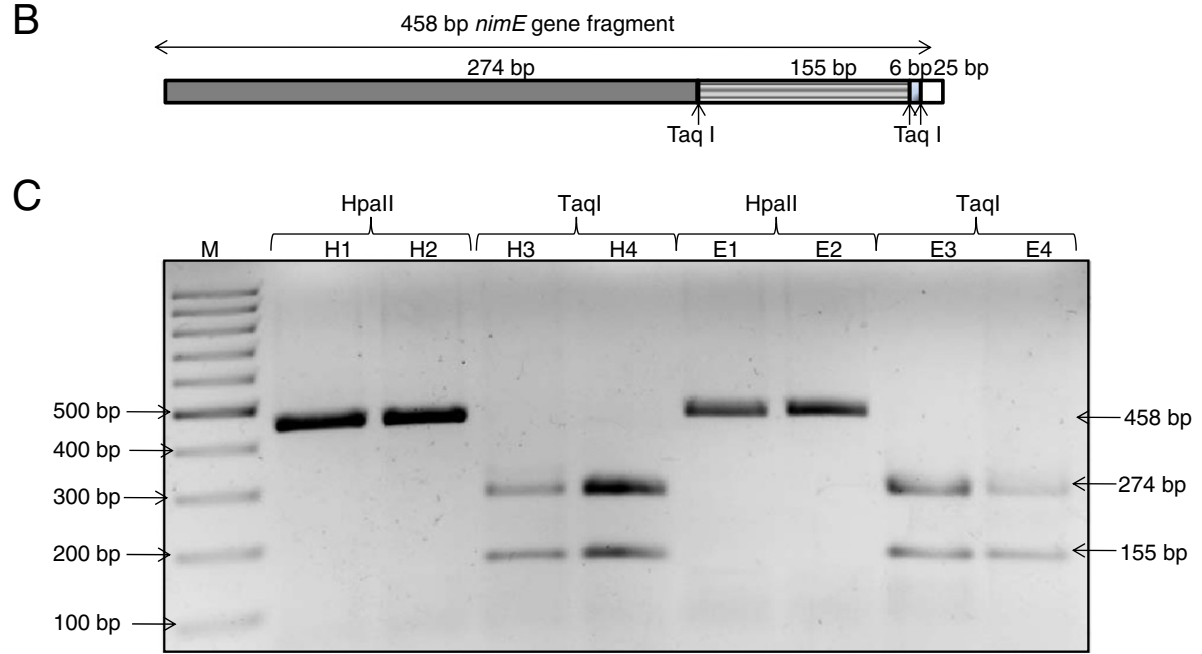

Figure 6 Detection and identification of nim gene in stool samples. (A) Detection of nim gene using nim gene specific primers. Lane 1 = Marker $100 \mathrm{bp}$, Lane 2 = clone of nim gene as positive control, Lane 3-5=DNA from stool samples from healthy volunteer, Lane 6-8= DNA from stool samples from E. histolytica positive patients and Lane $9=$ No template control PCR (B) Restriction map of Taql restriction sites in 458 bp nimE gene fragment. (C) Hpall does not digest nimE,where as digestion of nimE by Taql generates four fragment of 274 bp, 155 bp,6 bp and 25 bp. Lane 1 = Marker 100 bp, Lane H1, H2, E1 and E2 show RFLP profile of PCR product digested with Hpall; Lane H3, H4, E3 and E4 show RFLP profile of PCR product digested with Taql. H1-H4, DNA from stool samples of Healthy volunteers and E1-E4 are DNA from stool samples of E. histolytica positive patients.

balance in population of different bacterial genera of gut is needed for healthy colon.

The C. leptum subgroup and C. coccoides subgroup are one of the most predominant populations of human fecal microflora which contains a large number of butyrateproducing bacteria $[35,36]$. Butyrate is a SCFA (Short chain fatty acids) having a strong effect on the cell cycle and acts as anti-inflammatory molecule in the gut. Effects on mucosal defense include improved tight junction assembly, antimicrobial secretion and mucin expression [37]. The decrease in population of members of C. leptum subgroup and $C$. coccoides subgroup observed here leads to decrease in the production of SCFA and hence renders the host more susceptible for future infections.

The genus Lactobacillus comprises a large heterogenous group of low $\mathrm{G}+\mathrm{C}$ gram positive, non sporulating, anaerobic bacteria belonging to phylum Firmicutes. Lactobacilli are known to fortify epithelial barrier by various mechanism such as induction of mucin secretion, enhancement of tight-junction functioning, upregulation of cytoprotective heat shock proteins and prevention of apoptosis of epithelial cells [38]. Probiotic strains of Lactobacillus are known to prevent infectious diarrhea, antibiotic associated diarrhea and diarrhea in children who are unusually more susceptible as a result of poor nutrition, impaired immune status or frequent exposure to pathogens [39]. We observed significant decrease in population of Lactobacillus in gut flora of $E$. histolytica positive patients as compared to that of healthy individuals that support our earlier observation made by semi quantitative method [1].

Methanobrevibacter smithii is the dominant archaeon in human gut that affects the specificity and efficiency of bacterial digestion of dietary polysaccharides, thereby influencing host calorie harvest and adiposity [40]. It has been suggested that the low and variable prevalence of Methanobrevibacter smithii and Methanosphaera stadtmanae DNA in human stool contrasts with the paramount role of these methanogenic archaea in digestion processes and hypothesized that this contrast is a consequence of the inefficiencies of current protocols for archaea DNA extraction [41]. In our samples prevalence of M. smithii in healthy individuals stool samples was $27.27 \%$ and that was further reduced to $11.7 \%$ in $E$. histolytica positive samples. Real-time analysis shows no significant alteration in population of M. smithii. Variation in the loads of M. smithii under different pathophysiological condition such as during amebiasis has not been reported so far.

Suphate reducing bacteria (SRB) are a group of non spore forming, gram negative, dissimilatory sulphate reducing, anaerobic bacteria. SRB can be isolated from the intestinal tract of humans and various environmental sources. Intestinal SRB's growth and resultant hydrogen 
sulfide production have been implicated to damage the gastrointestinal tract and thereby contribute to chronic intestinal disorders [42]. Desulfovibrio fairfieldensis and $D$. desulfuricans have been associated with incidence of bacteremia and $D$. vulgaris has been associated with intra-abdominal infections [43]. The prevalence of Sulphate reducing bacteria was $36.36 \%$ in healthy and $11.7 \%$ in amoebic individuals stool samples. However, the change was not statistically significant.

The genus Campylobacter is notorious for causing gastroenritis by $C$. jejuni but uncultured Campylobacter species e.g. Campylobacter hominis whose role is not clear yet, do exist in lower gastrointestinal tract of healthy humans [44]. We observed significant decrease in population of Campylobacter in E. histolytica positive individual as compared to healthy individuals. As our primers were genus specific, so decrease in Campylobacter was genera specific and not species specific. Significant increase in the population of Campylobacter has been observed in IBD [21] but we did not find the same trend in amoebic patients.

Several species of Bacteroides are known to harbor nim genes e.g. B. fragilis, B. distasonis, B. thetaiotaomicron, $B$. vulgatus, $B$. ovatus but wide differences in MIC values of metronidazole are observed, ranging from 1.5 to $>256 \mathrm{mg} / \mathrm{L}$ and some are also found above the therapeutic breakpoint of $16 \mathrm{mg} / \mathrm{L}$ [45].Though the population of Bacteroides is decreased significantly in $E$. histolytica positive patients however we have observed high copy no. of nimE gene in the same. We attribute this increase to the presence of plasmid coded nimE gene as has been observed earlier in Veillonella sp. [46]. Future analyses that target specific members of the $\mathrm{Bac}$ teroides group will shed further light on the species involved in the expansion of nimE gene. In 2006, Rani et al. reported presence of nim gene in stool samples of amebic individuals but not in healthy individuals [1] but our result show high prevalence rate of nim gene even in healthy individuals irrespective of the disease. However in a hospital based study carried out in Greece revealed low level of prevalence of nim gene in isolates of different anaerobic bacterial species from hospitalized patients [47]. Though the presence of nim gene in gut of healthy north Indian population is shocking but this may be explained due to easy over the counter drug availability in India. Results on healthy individuals undergoing Satronidazole treatment indicate that nimE gene copy number does not show significant reduction. It can therefore be assumed that nimE gene harboring Bacteroides probably cause inactivation of nitroimidazole drug and thereby reduce the bioavailability of drug to the parasite and hence may help in sustaining the infection.

\section{Conclusion}

The metabolic activities of the predominant gut flora have a significant effect on the health of the human colon. The current findings of depleted populations of metabolically important bacteria like Bacteroides, $C$. leptum and C. coccoides sub groups, Lactobacillus sp., Eubacterium sp., and Campylobacter sp. add to our knowledge of the changes in the GI tracts of amebic patients. Such changes in bacterial population in the normal microbiota could have considerable consequences in terms of functional potential of gut flora and could result in metabolic conditions favorable for the establishment of opportunistic pathogens (e.g. Clostridium difficile). However, our study cannot conclude that observed changes in the gut flora is the cause or effect of the infection or the effect of dysenteric mechanism per se by the parasite. Our findings could potentially guide implementation of dietary/probiotic interventions that impact the gut microbiota and improve GI health in individuals infected with Entamoeba histolytica.

\section{Additional file}

Additional file 1: Real time analysis of population of (A) Methanobrevibacter in Healthy vs $\mathrm{E}$. histolytica positive samples (B) Sulphur reducing bacteria in Healthy vs E. histolytica positive sample. $\mathrm{P}$ value $=.05$ or below was considered significant. $\mathrm{Cl}$ stands for confidence interval.

\section{Abbreviations}

SRB: Sulphur reducing bacteria; RFLP: Restriction fragment length polymorphism; MUC2: Mucin 2; SCFA: Short-chain.

\section{Competing interests}

We declare that no competing interests exist among the authors

\section{Authors' contributions}

JP conceived and coordinated the study. AKV carried out the bacterial quantification experiments. AKV and RV conducted the copy number calculation experiments. JP and AKV drafted the manuscript and conducted the statistical analysis. VA made the diagnosis of the patients, interpretation of data and collaborated in collection of the samples. All authors read and approved the final manuscript.

\section{Acknowledgement}

This study was supported by a research grant to JP from the Department of Biotechnology, Government of India. AKV is grateful to the Indian Council of Medical Research, New Delhi, India and RV to University Grants Commission, New Delhi for Research fellowships. We gratefully acknowledge the subjects who participated in this study.

\section{Author details}

${ }^{1}$ School of Life Sciences, Jawaharlal Nehru University, New Delhi, India. ${ }^{2}$ Department of Gastroenterology, All India Institute of Medical Sciences, New Delhi, India.

Received: 5 November 2011 Accepted: 30 July 2012

Published: 22 August 2012

\section{References}

1. Rani R, Murthy RS, Bhattacharya S, Ahuja V, Rizvi MA, Paul J: Changes in Bacterial profile during amebiasis: Demonstration of anaerobic bacteria in ALA pus samples. Am J Trop Med Hyg 2006, 75:880-885. 
2. Jia W, Li H, Zhao L, Nicholson JK: Gut microbiota: a potential new territory for drug targeting. Nat Rev Drug Discov 2008, 7:123-129.

3. Whitman WB, Coleman DC, Wiebe WJ: Prokaryotes: The unseen majority. Proc Natl Acad Sci USA 1998, 95:6578-6583.

4. Sonnenburg JL, Angenent LT, Gordon J: Getting a grip on things: how do communities of bacterial symbionts become established in our intestine? Nat Immunol 2004, 5:569-573.

5. Xu J, Gordon Jl: Honor thy symbionts. Proc Natl Acad Sci USA 2003, 100:10452-10459.

6. Guarner F: Enteric flora in health and disease. Digestion 2006, 73(suppl 1):5-12

7. O'Hara AM, Shanahan F: The gut flora as a forgotten organ. EMBO Rep 2006, 7:688-693.

8. Ley RE, Peterson DA, Gordon Jl: Ecological and Evolutionary Forces Shaping Microbial Diversity in the Human Intestine. Cell 2006, 124:837-848.

9. Haque R, Huston CD, Hughes M, Houpt E, Petri WA Jr: Amoebiasis. N Engl J Med 2003, 348:1565-1573.

10. Mirelman D: Ameba-bacterium relationship in amoebiasis. Microbio/ Rev 1987, 51:272-284.

11. Mukherjee C, Clark CG, Lohia A: Entamoeba Shows Reversible Variation in Ploidy under Different Growth Conditions and between Life Cycle Phases. PLoS Negl Trop Dis 2008, 2:e281.

12. Simon GL, Gorbach SL: Intestinal flora in health and disease. Gastroenterology 1984, 86:174-193.

13. Leiros HKS, Kozielski-Stuhrmann S, Kapp U, Terradot L, Leonard GA, McSweeney SM: Structural Basis of 5-Nitroimidazole Antibiotic Resistance. J Biol Chem 2004, 279:55840-55849.

14. Trinh S, Reysset G: Detection by PCR of the nim Genes Encoding 5Nitroimidazole Resistance in Bacteroides spp. J Clin Microbiol 1996, 34:2078-2084.

15. Petri WA Jr: Amebiasis. Current treatment options in Infectious diseases 2003, 5:269-272.

16. Knight WB, Hiatt RA, Cline BL, Ritchie LS: A Modification of the FormolEther Concentration Technique for Increased Sensitivity in Detecting Schistosoma Mansoni Eggs. Am J Trop Med Hyg 1976, 25:818-823.

17. Srivastva S, Bhattacharya S, Paul J: Species and strain-specific probes derived from repetitive DNA for distinguishing Entamoeba histolytica and Entamoeba dispar. Exp Parasitol 2005, 110:303-308.

18. Niesters HGM: Clinical virology in realtime. J Clin Virol 2002, 25:S3-S12.

19. Stubbs SLJ, Brazier J, Talbot PR, Duerden BI: PCR-Restriction Fragment Length Polymorphism Analysis for Identification of Bacteroides spp. and Characterization of Nitroimidazole Resistance Genes. J Clin Microbiol 2000, 38:3209-3213.

20. Whelan JA, Russell NB, Whelan MA: A method for the absolute quantification of cDNA using real-time PCR. J Immunol Methods 2003, 278:261-269.

21. Verma R, Verma AK, Ahuja $V$, Paul J: Real-Time Analysis of Mucosal Flora in Patients with Inflammatory Bowel Disease in India. J Clin Microbiol 2010, 48:4279-4282

22. Altschul SF, Gish W, Miller W, Myers EW, Lipman DJ: Basic local alignment search tool. J Mol Biol 1990, 215:403-410.

23. Galvan-Moroyoqui JM, Domı/nguez-Robles MDC, Franco E, Meza I: The Interplay between Entamoeba and Enteropathogenic Bacteria Modulates Epithelial Cell Damage. Plos Neg Trop Dis 2008, 2:e266.

24. Seksik P, Rigottier-Gois L, Gramet G, Sutren M, Pochart P, Marteau P, Jian R, Doré J: Alterations of the dominant faecal bacterial groups in patients with Crohn's disease of the colon. Gut 2003, 52:237-242.

25. Tannock GW, Munro K, Harmsen HJM, Welling GW, Smart J, Gopal PK: Analysis of the fecal microflora of human subjects consuming a probiotic product containing Lactobacillus rhamnosus DR20. Appl Environ Microbiol 2000, 66:2578-2588.

26. Bhattacharaya A, Anand MT, Paul J, Yadav N, Bhattacharaya S: Molecular Changes in Entamoeba histolytica in Response to Bacteria. J Euk Microbiol 1998, 45:28S-33S.

27. Hartman AL, Lough DM, Barupal DK, Fiehn O, Fishbein T, Zasloff M, Eisen JA: Human gut microbiome adopts an alternative state following small bowel transplantation. Proc Natl Acad Sci USA 2009, 106:17187-17192.

28. Hooper LV, Xu J, Falk PG, Midtvedt T, Gordon J: A molecular sensor that allows a gut commensal to control its nutrient foundation in a competitive ecosystem. Proc Natl Acad Sci USA 1999, 96:9833-9838.
29. Kanauchi O, Fujiyama Y, Mitsuyama K, Araki Y, Ishii T, Nakamura T, Hitomi Y, Agata K, Saiki T, Andoh A, Toyonaga A, Bamba T: Increased growth of Bifidobacterium and Eubacterium by germinated barley foodstuff, accompanied by enhanced butyrate production in healthy volunteers. Int J Mol Med 1999, 3:175-179.

30. Simmering $\mathrm{R}$, Kleessen B, Blaut M: Quantification of the FlavonoidDegrading Bacterium Eubacterium ramulus in Human Fecal Samples with a Species-Specific Oligonucleotide Hybridization Probe. Appl Env Microbiol 1999, 65:3705-3709.

31. Hopkins MJ, Macfarlane GT: Changes in predominant bacterial populations in human faeces with age and with Clostridium difficile infection. J Med Microbiol 2002, 51:448-454.

32. Fukuda S, Toh H, Hasel K, Oshima K, Nakanishi Y, Yoshimura K, Tobe T, Clarke JM, Topping DL, Suzuki T, Taylor TD, Itoh K, Kikuchi J, Morita H, Hattori M, Ohno H: Bifidobacteria can protect from enteropathogenic infection through production of acetate. Nature 2011, 469:543-547.

33. Leitch EC, Walker AW, Duncan SH, Holtrop G, Flint HJ: Selective colonization of insoluble substrates by human faecal bacteria. Env Microbiol 2007, 9:667-679.

34. Lidell ME, Moncada DM, Chadee K, Hansson GC: Entamoeba histolytica cysteine proteases cleave the MUC2 mucin in its C-terminal domain and dissolve the protective colonic mucus gel. Proc Natl Acad Sci USA 2006, 103:9298-9303.

35. Pryde SE, Duncan SH, Hold GL, Stewart CS, Flint HJ: The microbiology of butyrate formation in the human colon. FEMS Microbiol Lett 2002, 217:133-139.

36. Robert C, Bernalier-Donadille A: The cellulolytic microflora of the human colon: evidence of microcrystalline cellulose-degrading bacteria in methane-excreting subjects. FEMS Microbiol Eco 2003, 46:81-89.

37. Willing BP, Russell SL, Finlay BB: Shifting the balance: antibiotic effects on host-microbiota mutualism. Nat Rev Microbiol 2011, 9:233-243.

38. Lebeer S, Vanderleyden J, De Keersmaecker SCJ: Genes and Molecules of Lactobacilli supporting Probiotic Action. Microbiol Mol Biol Rev 2008, 72:728-764

39. Van Neil CW, Feudtner C, Garrison MM, Christakis DA: Lactobacillus therapy for acute infectious diarrhea in children:A meta analysis. Pediatrics 2002, 109:678-684.

40. Samuel BS, Hansen EE, Manchester JK, Coutinho PM, Henrissat B, Fulton R, Latreille P, Kim K, Wilson RK, Gordon Jl: Genomic and metabolic adaptations of Methanobrevibacter smithii to the human gut. Proc Natl Acad Sci USA 2007, 104:10643-10648.

41. Dridi B, Henry M, Khechine AE, Raoult D, Drancourt M: High Prevalence of Methanobrevibacter smithii and Methanosphaera stadtmanae detected in the Human Gut Using an Improved DNA Detection Protocol. PLoS One 2009, 4:e7063.

42. Deplancke B, Hristova KR, Oakley HA, McCracken VJ, Aminov R, Mackie Rl, Gaskins HR: Molecular ecological analysis of the succession and diversity of Sulphate reducing bacteria in mouse gastrointestinal tract. Appl Env Microbiol 2000, 66:2166-2174.

43. Goldstein EJC, Citron DM, Peraino VA, Cross SA: Desulfovibrio desulfuricans Bacterimia and Review of Human Desulfovibrio infections. J Clin Microbiol 2003, 41:2752-2754.

44. Lawson AJ, Linton D, Stanley J: 16 s rRNA gene sequences of 'Candidatus Campylobacter horninis', a novel uncultivated species, are found in the gastrointestinal tract of healthy humans. Microbiol 1998, 144:2063-2071.

45. Gal M, Brazier JS: Metronidazole resistance in Bacteroides spp. carrying nim genes and the selection of slow-growing metronidazole-resistant mutants. J Antimicrob Chemother 2004, 54:109-116.

46. Marchandin $\mathrm{H}$, Jean-Pierre $\mathrm{H}$, Campos J: nimE Gene in a MetronidazoleSusceptible Veillonella sp Strain. Antimicrob Agents Chemother 2004 48:3207-3208

47. Katsandri A, Avlamis A, Pantazatou A, Houhoula DP, Papaparaskevas J: Dissemination of nim-class genes, encoding nitroimidazole resistance, among different species of Gram-negative anaerobic bacteria isolated in Athens, Greece. J Antimicrob Chemother 2006. doi:10:1093/jac/dkl285.

doi:10.1186/1471-2180-12-183

Cite this article as: Verma et al:: Real-time analysis of gut flora in Entamoeba histolytica infected patients of Northern India. BMC Microbiology 2012 12:183. 